\title{
Drosophila Do Not Skew Offspring Sex Ratio As Predicted by Trivers and Willard (Diptera: Drosophilidae)
}

\author{
Russell L. Burke ${ }^{1}$ and Lance E. Little ${ }^{2}$ \\ Accepted February 10, 1994; revised March 1, 1994
}

Based on both previously published literature and results reported here, it appears that Drosophila melanogaster meet the explicit assumptions of the Trivers and Willard offspring sex allocation model. However, contrary to the model's predictions, offspring sex ratio was not significantly affected when we manipulated factors that influence offspring quality. We suggest that contrary to implicit predictions of offspring sex ratio models, Drosophila may lack the genetic plasticity to readily alter sex ratio.

KEY WORDS: Drosophila; sex ratio; life history; optimality model.

\section{INTRODUCTION}

Trivers and Willard (1973) suggested that under certain conditions, optimally behaving organisms may adjust the sex ratio of their offspring as a function of maternal condition. In particular, Trivers and Willard hypothesized that mothers whose offspring are likely to be in above average condition (relative to other offspring) are likely to have male-biased offspring sex ratios, whereas if the offspring were likely to be in relatively poor condition, offspring sex ratio would be female biased. Their hypothesis was based on three explicit assumptions: (1) The mother's condition during parental investment (PI) and the offspring condition at the end of PI are positively correlated; (2) variance in the condition of the offspring at the end of PI tends to endure into adulthood; and (3) for polygynous species, a son in above-average condition is likely to have higher repro-

\footnotetext{
'Museum of Zoology and Department of Biology, University of Michigan, Ann Arbor, Michigan 48109-1079.

${ }^{2} 2863$ East Grand River Road, Bancroft, Michigan 48414.
} 
ductive fitness than a daughter in similar condition, while a daughter in poor condition will have higher reproductive fitness than a son in similar condition.

A variety of tests and some support for Trivers and Willard's model have been reported for many species of mammals (for examples see Frank, 1990). However, while the model's predictions have been tested in some Hymenoptera (e.g., Longair, 1981; Wellings et al., 1986; King, 1988), tests of diploid invertebrates have not been reported. Here we address this question with the fruit fly, Drosophila melanogaster, a polygynous species for which there is reasonably well-documented support for each of Trivers and Willard's three assumptions (see below).

To test for the tendency of female Drosophila melanogaster to bias offspring sex ratios, we tested the applicability of Trivers and Willard's assumptions, as well as the predictions of their model. We examined the impact of variation in maternal condition on offspring body size and therefore future performance. This was accomplished by examining the effects of two factorsmaternal body size and, by manipulating female densities, the ability of females to oviposit in sites that minimize larval competition for resources. On the basis of previous Drosophila literature and the Trivers and Willard hypothesis, we predicted that as female size increased, egg size, mean individual offspring mass, and offspring sex ratio (percentage sons) would increase. Also, we predicted that as adult density decreased, mean individual offspring mass and offspring sex ratio would increase.

\section{MATERIALS AND METHODS}

A wild population of Drosophila melanogaster originally collected in 1991 in Ann Arbor, Michigan, was expanded into 20 bottles and maintained on a complete high-yeast glucose medium (Ashburner, 1989) at $25^{\circ} \mathrm{C}$. As the flies eclosed (emerged from pupal case), virgin females and males were collected twice a day for 7 days and stored separately. At the end of this period, females were sorted by eye into three size classes. Head-body lengths of all "small" and "large," and a random sample of "medium," females were recorded.

Six experimental treatments were prepared: populations of small, medium, and large females, each with four females per population, plus additional treatments of medium females at densities of 1,10 , and 20 females per population (see Table I). Each treatment had three to five replicate populations. Populations were maintained in vials $(28-\mathrm{mm}$ diameter and $95-\mathrm{mm}$ length with $10 \mathrm{ml}$ of high-yeast glucose food) and transferred to new vials for egg collection every $12 \mathrm{~h}$ for 5 days, resulting in a total of 10 vials for each population. Length measurements of a random sample of eggs from each initial vial were recorded. After transfer of a population to a new vial, vials with eggs were stored at $25^{\circ} \mathrm{C}$ for 13 days (i.e., until 3 days after the eclosion of the first offspring). All eclosed 


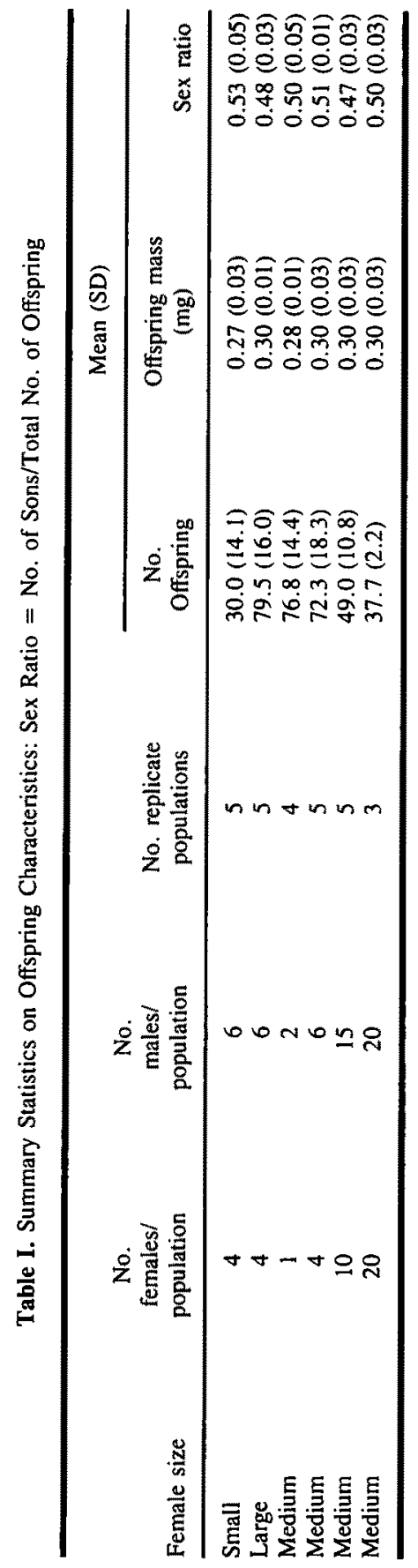


males and females from each vial were removed and counted when the populations were 13,16, and 18 days old. Dry body mass of flies from each run of replicates from the first two egg collection was recorded.

The hypotheses tested here predict that differences between treatments will be in specific directions, therefore rather than standard ANOVA procedures we used isotonic regression analysis. This one-tailed analysis results in a significant increase in statistical power over two-tailed ANOVA (Gaines and Rice, 1990).

\section{RESULTS}

Due to generally small sample sizes, data were plotted as frequency distributions and visually inspected for normality; all distributions except offspring sex ratios appeared approximately normal. Sex ratio values (number of males/ total number of offspring) were therefore arc-sign transformed (Sokal and Rohlf, 1969); the resulting data appeared normal. First, we tested whether our crude separation of parental females into size classes was effective; we found that the "small" parental females were significantly smaller than the "medium" females (Table II; $t=3.25$, $\mathrm{df}=38, P<0.001$ ), and "large" were significantly larger than medium females $(t=14.05, \mathrm{df}=38, P<0.001)$. Similarly, eggs from large females were significantly bigger than those from either medium (Table II; $t=1.8, \mathrm{df}=84, P<0.025$ ) or small females $(t=1.76, \mathrm{df}=64$, $P<0.025)$. Offspring sex ratio was analyzed by isotonic regression with female size and female density as independent variables, with directions tested as indicated in our predictions (see above). Neither was significant (Table I; $\epsilon_{3}^{2}<$ $0.01, P=0.68$, and $\epsilon_{4}^{2}=0.007, P=0.64$, respectively). Similarly, we did not find significant relationships when we analyzed offspring mass with female size and female density (Table $\mathrm{I}, \epsilon_{3}^{2}=0.01, P=0.09$, and $\epsilon_{4}^{2}=0.116, P=$ 0.23 , respectively). We also tested whether the number of offspring/female parent was affected by either female density or size. Here we used one-way

Table II. Summary Statistics on Female Size and Egg Size: Egg Size Measured in Uncalibrated Ocular Units

\begin{tabular}{llc}
\hline & \multicolumn{2}{c}{ Mean (SD) } \\
\cline { 2 - 3 } Size class & $\begin{array}{c}\text { Female size } \\
(\mathrm{mm})\end{array}$ & Egg size \\
\hline Small & $2.25(0.34)$ & $8.3(0.57)$ \\
Medium & $2.50(0.01)$ & $8.4(0.69)$ \\
Large & $3.08(0.18)$ & $8.6(0.54)$ \\
\hline
\end{tabular}


ANOVA because we did not make prior predictions. Increasing adult female density (among medium-sized females) had a significant negative effect (Table I; $F_{3,12}=14.06, P<0.001$ ), and increasing adult female size a significant positive effect (Table I; $F_{2.11}=10.13, P=0.003$ ), on the number of offspring/ female parent.

\section{DISCUSSION}

The three explicit assumptions of Trivers and Willard's (1973) hypothesis involve the "condition" of individuals, relative to others in the same population. Trivers and Willard suggested several ways to rank maternal condition, including parity, litter size, and measures of environmental quality. Other workers have successfully used maternal social rank (i.e., Clutton-Brock et al., 1986) and maternal body fat (i.e., Kucera, 1991). We assumed that the parameters we used were important because they appear to be major factors influencing offspring size and, therefore, presumably both parental and offspring fitness. However, we found no association between offspring sex ratio and either maternal body size or larval density.

Trivers and Willard's first assumption was that offspring condition during parental investment (PI) tends to be positively correlated with maternal condition. For Drosophila, PI is limited to the time from oogenesis until complete egg formation [about $79 \mathrm{~h}$ (David and Merle, 1968)]. Numerous studies have shown that larvae from eggs oviposited where food is abundant and where there is little competition will be well nourished and generally larger. For example, as larval density increases, larval size decreases (Chiang and Hodson, 1950), as do pupal size (Chiang and Hodson, 1950) and mass (Sang, 1949). The effect of increased larval and/or parental density is also accompanied by a decrease in the proportion of larvae that pupate (Chiang and Hodson, 1950; Barker and Podger, 1970; Sang 1949) and survive into adulthood (Barker, 1973; Chiang and Hodson, 1950; Sang, 1949). Sokoloff (1955) has shown similar reductions in preadult and adult viability in three sibling species of Drosophila. Barker (1973) found that as parental densities increase, the average number of progeny per female decreases. He concluded that, for D. melanogaster, this was due to the combined effects of decreased female fecundity and reduced larval and pupal viability due to larval crowding.

We interpret our finding that maternal body size was positively associated with egg size as partial support for Trivers and Willard's first assumption. To substantiate this it would be necessary to show that egg size is a good indication of offspring condition. However, the functional relationship between egg size and any subsequent measures of survivorship or reproduction is unknown. We found that the number of offspring per female decreased as the density increased and also as the female body size decreased; clearly, the number of offspring is 
associated with the female condition. We do not know whether this was a result of reduced fecundity, or decreased larval survivorship, or both.

Trivers and Willard's second assumption was that differences in offspring condition at the end of PI tend to endure into adulthood. Pupal size in Drosophila is strongly correlated with subsequent adult body size (Chiang and Hodson, 1950), and after eclosure, body size in Drosophila is relatively fixed. As larval density is increased, adult mass decreases (Economos et al., 1981; Barker, 1973; Barker and Podger, 1970; Sang, 1949; Miller and Thomas, 1958), as does adult body size (Chiang and Hodson, 1950). However, although our high-density treatments clearly resulted in a reduction in the number of offspring per female, offspring size was not reduced as in these previous studies. Apparently, higher densities than those we used are required for a reduction in larval size to occur.

Trivers and Willard's third assumption was that there are differential benefits in reproductive success between sons and daughters if both are provided with the same level of parental care. Support for this assumption in Drosophila comes mainly from data showing that various condition measures are strongly associated with male mating success. Males have considerably greater variation in reproductive success, and this result is due largely to variation in the number of matings achieved (Bateman, 1948). In fact, Duncan (1930) found that vigorous males may father as many as 10,000 to 14,000 offspring, while it is unlikely that any females can lay anywhere near this many eggs. In wild populations, larger males are more likely to obtain mates (Partridge et al., 1987b; Markow, 1988; Taylor and Kekic, 1988).

Partridge and Farquhar (1983) suggest that the advantage of a larger male is twofold-larger males live longer and, at least during their first 2 weeks as adults, inseminate more females than do smaller males. They also showed that larger males have higher mating speeds. Partridge et al. (1987a) found that larger males court longer, produce louder and longer courtship song, and search a larger area in search of potential mates. They also suggested that, because females move faster than males during courtship, larger males may be better able to keep up with the female. Larger males are also more likely to win aggressive encounters (Markow, 1988; Dow and von Schilcher, 1975). Other research has reinforced these general conclusions (Pitnick, 1991; Partridge et al., 1987a; Ewing, 1964; Markow, 1986; Partridge, 1988; Partridge and Farquhar, 1983; Ewing, 1961). On the basis of our own and these other studies, it appears that there is reasonable support for Trivers and Willard's third assumption.

A potential criticism of the conclusion that larger males have higher reproductive success in wild populations is that mating success is instead frequency dependent. Some laboratory research on various Drosophila species has suggested the importance of a "rare-male" mating advantage [for a general review see Ehrman and Probber (1978); in melanogaster, Petit (1951, 1954, 1958)], 
and if true, this phenomenon should be considered when interpreting experiments that relate male size to mating success. However, recent reports have shown that rare-male success can occur spuriously (Knoppien, 1987; Markow, 1980; Markow et al., 1980; Spiess, 1982; Gromko et al., 1980), and this phenomenon is now thought to be relatively unimportant in wild populations.

Although the assumptions outlined by Trivers and Willard (1973) appear to be met in $D$. melanogaster, we found no evidence of the predicted offspring sex ratio bias. While there are many possible explanations for this, we suggest two as particularly important. First, it may be that the condition indices we measured were not relevant in this species or that their importance was swamped by other, more important factors influencing reproductive fitness. Second, the model may be appropriate but fail to predict Drosophila behavior for a more fundamental reason. A common but usually implicit assumption of most optimality models is that sufficient genetic variability exists such that organisms can readily evolve characteristics that maximize fitness. In contrast, though it might be advantageous for individuals to do so, Drosophila may simply not have the mechanistic ability to alter offspring sex ratio as a function of maternal condition. Other workers (Toro and Charlesworth, 1982; Curtsinger, 1981; Falconer, 1954) have reported poor success in producing strongly sex ratio-biased lines in controlled Drosophila breeding experiments. These types of results have resulted in the suggestion (i.e., Toro and Charlesworth, 1982; Williams, 1979) that this inability may be generally true of all diploid organisms. However, the mammalian studies cited above and those in Frank (1990) demonstrate that adaptive sex ratio biasing does apparently occur in some diploid species. Our results imply that if, in fact, selective pressures have existed that would favor development of the ability to adaptively alter offspring sex ratio in Drosophila, they have been insufficient to result in the evolution of this behavior.

\section{ACKNOWLEDGMENTS}

We thank H. LeVine for supplying the original fly stock and D. C. Otteson from the Drosophila Research Facility of The University of Michigan for invaluable advice and use of laboratory facilities. James Birch, Jae Choe, Ray Huey, Ron Nussbaum, and two anonymous reviewers made helpful comments on early versions of the manuscript. Steve Gaines generously provided a copy of his isotonic regression program, and The Department of Biology, University of Michigan, provided partial support for this work.

\section{REFERENCES}

Ashbumer, M. (1989). Drosophila: A Laboratory Handbook, Cold Spring Harbor Laboratory Press, Cold Spring Harbor, NY. 
Barker, J. S. F. (1973). Adult population density, fecundity and productivity in Drosophila melanogaster and Drosophila simulans. Oecologia 11: 83-92.

Barker, J. S. F., and Podger, R. N. (1970). Interspecific competition between Drosophila melanogasier and Drosophila simulans: Effects of larval density on viability and adult body size. Ecology 51: 170-189.

Bateman, A. J. (1948). Intra-sexual selection in Drosophila. Heredity 2: 349-368.

Chiang, H. C., and Hodson, A. C. (1950). An analytical study of population growth in Drosophila melanogaster. Ecol. Monogr. 20: 173-206.

Clutton-Brock, T. H., Aldon, S. D., and Guinness, F. E. (1986). Great expectations: Dominance, breeding success, and offspring sex ratios in red deer. Anim. Behav. 34: 460-471.

Curtsinger, J. W. (1981). Artificial selection on the sex ratio in Drosophila pseudoobscura. $J$. Hered. 72: 377-381.

David, J. R., and Merle, J. (1968). A reevaluation of the duration of egg chamber stages in oogenesis of Drosophila melanogaster. Dros. Info. Serv. 43: 122-123.

Dow, M. A., and von Schilcher, F. (1975). Aggression and mating success in Drosophila melanogaster. Nature 254: 511-512.

Duncan, F, N. (1930). Some observations on the biology of the male Drosophila melanogaster. Am. Nat. 44: 545-551.

Economos, A. C., Lints, C. V., and Lints, F. A. (1981). On the mechanisms of the effects of larval density and temperature on Drosophila development. In Lakovaara, S. (ed.), Advances in Genetics, Development, and Evolution of Drosophila, Plenum Press, New York, pp. 149164.

Ehrman, L., and Probber, J. (1978). Rare Drosophila males: The mysterious matter of choice. Am. Sci. 66: 216-222.

Ewing, A. (1961). Body size and courtship behavior in D. melanogaster. Anim. Behav. 9: 93-99.

Ewing, A. W. (1964). The influence of wing area on the courtship of Drosophila melanogaster. Anim. Behav. 12: 316-320.

Falconer, D. S. (1954). Selection for sex ratio in mice and Drosophila. Am. Nat. 88: 385-397.

Frank, S. A. (1990). Sex allocation theory for birds and mammals. Annu. Rev. Ecol. System. 21: 13-55.

Gaines, S. D., and Rice, W. R. (1990). Analysis of biological data when there are ordered expectations. Am. Nat. 135: 310-317.

Gromko, M. H., Sheehan, K., and Richmond, R. C. (1980). Random mating in two species of Drosophila. Am. Nat. 115: 467-479.

King, B. H. (1988). Sex ratio manipulation in response to host size by the parasitoid wasp Spalangia cameroni-A laboratory study. Evolution 42: 1190-1198.

Knoppien, P. (1987). Rare-male mating advantage: An artifact caused by differential storage conditions? Behav. Genet. 17: 409-425.

Kucera, T. E. (1991), Adaptive variation in sex ratios of offspring in nutritionally stressed mule deer. J. Mammal. 72: 745-749.

Longair, R. W. (1981). Sex-ratio variation in xylophilous aculeate Hymenoptera. Evolution 35: 597-600.

Markow, T. A. (1980). Rare male advantages among Drosophila of the same laboratory strain. Behav. Genet. 10: 553-556.

Markow, T. A. (1986). Genetic and sensory basis of sexual selection in Drosophila. In Huettel, M. D. (ed.), Evolutionary Genetics of Invertebrate Behavior, Plenum Press, New York, pp. 89-95.

Markow, T. A. (1988). Reproductive behavior of Drosophila melanogaster and D. nigrospiracula in the field and in the laboratory. J. Comp. Psychol. 102: 169-173.

Markow, T. A., Richmond, R. C., Mueller, L., Sheer, I., Roman, S., Laetz, C., and Lorenz, L. (1980). Testing for rare male advantages among various Drosophila melanogaster genotypes. Genet. Res. Cambr. 35: 59-64.

Miller, R. S., and Thomas, J. L. (1958). The effects of larval crowding and body size on the longevity of aduit Drosophila melanogaster. Ecology 37: 118-125.

Partridge, L. (1988). Lifetime reproductive success in Drosophila. In Clutton-Brock, T. H. (ed.), Reproductive Success, University of Chicago Press, Chicago, pp. 11-23. 
Partridge, L., and Farquhar, M. (1983). Lifetime mating success of male fruit flies (Drosophila melanogaster) is related to their size. Anim. Behav. 31: 871-877.

Partridge, L., Ewing, A., and Chandler, A. (1987a). Male size and mating success in Drosophila melanogaster: The roles of male and female behavior. Anim. Behav. 35: 555-562.

Partridge, L., Hoffmann, A., and Jones, J. S. (1987b). Male size and mating success in Drosophila melanogaster and D. pseudoobscura under field conditions. Anim. Behav. 35: 468-476.

Petit, C. (1951). Le role de l'isolement sexual dans l'évolution des populations de Drosophila melanogaster. Bull. Biol. France Belg. 85: 392-418.

Petit, C. (1954). L'isolement sexual chez Drosophila melanogaster. Etude du mutant white et de son alle'lomorphe sauvage. Bull. Biol. France Belg. 88: 435-443.

Petit, C. (1958). Le déterminisme génétique et psycho-physologique de la compétition sexuelle chez Drosophila melanogaster. Bull. Biol. France Belg. 92: 248-329.

Pitnick, S. (1991). Male size influences mate fecundity and remating interval in Drosophila melanogaster. Anim. Behav. 41: 735-745.

Sang, J. H. (1949). The ecological determinants of population growth in Drosophila culture. III. Larval and pupal survival. Physiol. Zool. 22: 183-202.

Sokal, R. R., and Rohlf, F. J. (1969). Biometry: The Principles and Practice of Statistics in Biological Research, W. H. Freeman, San Francisco.

Sokoloff, A. (1955). Competition between sibling species of the pseudoobscura subgroup of Drosophila. Ecol. Monogr. 25: 387-409.

Spiess, E. B. (1982). Do female flies choose their mates? Am. Nat. 119: 675-693.

Taylor, C. E., and Kekic, V. (1988). Sexual selection in a natural population of Drosophila melanogaster. Evolution 42: 197-199.

Toro, M. A., and Charlesworth, B. (1982). An attempt to detect genetic variation in sex ratio in Drosophila melanogaster. Heredity 49: 199-209.

Trivers, R. L., and Willard, D. E. (1973). Natural selection of parental ability to vary sex ratio of offspring. Science 179: 90-92.

Wellings, P. W., Hart, P. J., and Morton, R. (1986). Primary sex ratio and differential progeny survivorship in solitary haplo-diploid parasitoids. Ecol. Entomol. 11: 341-348.

Williams, G. C. (1979). The question of adaptive sex ratio in outcrossed vertebrates. Proc. Roy. Soc. London B 205: 567-580. 